UDK $577.1: 61$

ISSN 1452-8258

J Med Biochem 39: 91-99, 2020

\title{
NLRP3 AND CARD8 POLYMORPHISMS INFLUENCE RISK FOR ASBESTOS-RELATED DISEASES
}

\author{
UTICAJ NLRP3 I CARD\& POLIMORFIZAMA NA BOLESTI U VEZI SA AZBESTOM
}

\author{
Alenka Franko ${ }^{1}$, Katja Goricar ${ }^{2}$, Viljem Kovac ${ }^{3}$, Metoda Dodic-Fikfak', Vita Dolzan ${ }^{2}$ \\ ${ }^{1}$ Clinical Institute of Occupational Medicine, University Medical Centre Ljubljana, Ljubljana, Slovenia \\ ${ }^{2}$ Pharmacogenetics Laboratory, Institute of Biochemistry, Faculty of Medicine, University of Ljubljana, \\ Ljubljana, Slovenia \\ ${ }^{3}$ Institute of Oncology Ljubljana, Ljubljana, Slovenia
}

\section{Summary}

Background: This study aimed to investigate the association between NLRP3 rs35829419 and CARD 8 rs2043211 polymorphisms and the risk of developing pleural plaques, asbestosis, and malignant mesothelioma (MM), and to study the influence of the interactions between polymorphisms and asbestos exposure on the risk of developing these diseases.

Methods: The case-control study included 416 subjects with pleural plaques, 160 patients with asbestosis, 154 subjects with MM and 149 subjects with no asbestos disease. The NLRP3 rs35829419 and CARD8 rs2043211 polymorphisms were determined using real-time PCRbased methods. In the statistical analysis, standard descriptive statistics was followed by univariate and multivariate logistic regression modelling.

Results: Asbestos exposure (medium and high vs low) was associated with the risk for each studied asbestos-related disease. An increased risk of pleural plaques was found for CARD 8 rs2043211 AT + TT genotypes (OR $=1.48,95 \%$ $\mathrm{Cl} 1.01-2.16, \mathrm{p}=0.042)$. When the analysis was performed for $M M$ patients as cases, and pleural plaques patients as controls, a decreased MM risk was observed for carriers of CARD 8 rs2043211 TT genotype (OR $=0.52$, $95 \% \mathrm{Cl} 0.27-1.00, \mathrm{p}=0.049)$. The interactions between NLRP3 rs35829419 and CARD 8 rs2043211 genotypes did not influence the risk of any asbestos-related disease. However, when testing interactions with asbestos exposure,
\end{abstract}

\section{Kratak sadržaj}

Uvod: Cilj ovog istraživanja bio je da se ispita povezanost između polimorfizama NLRP3 rs35829419 i CARD8 rs2043211 i rizika od razvoja pleuralnih plakova, azbestoze i malignog mezotelioma (MM) i da se prouči uticaj interakcija između polimorfizama i izloženosti azbestu na rizik od razvoja ovih bolesti.

Metode: Ova studija slučaja je uključivala 416 ispitanika sa pleuralnim plakovima, 160 pacijenata sa azbestozom, 154 ispitanika sa MM i 149 ispitanika bez azbestne bolesti. Polimorfizmi NLRP3 rs35829419 i CARD8 rs2043211 su određivani pomoću metoda zasnovanih na PCR u realnom vremenu. U statističkoj analizi, standardnu deskriptivnu statistiku pratilo je univarijantno i multivarijantno logističko regresiono modeliranje.

Rezultati: Izloženost azbestu (srednja i visoka u odnosu na nisku) bila je povezana sa rizikom za svaku proučavanu bolest povezanu sa azbestom. Povećan rizik od pleuralnih plakova je ustanovljen za CARD 8 rs2043211 AT + TT genotipove $(O R=1,48,95 \% \mathrm{Cl} 1,01-2,16, p=0,042)$. Kada je obavljena analiza za pacijente sa MM, kao i za pacijente sa pleuralnim plakovima kao kontrolne slučajeve, primećen je smanjeni MM rizik za nosioce CARD 8 rs2043211 TT genotipa (OR $=0,52,95 \% \mathrm{Cl} 0,27-1,00, p=0,049)$. Interakcije između genotipova NLRP3 rs35829419 CARD 8 rs2043211 nisu uticale na rizik od bilo koje bolesti povezane sa azbestom. Međutim, kada su testirane interakcije sa izloženošću azbestu, ustanovljen je smanjen rizik

Address for correspondence:

Vita Dolzan

Pharmacogenetics Laboratory, Institute of Biochemistry

Faculty of Medicine, University of Ljubljana

2 Vrazov trg, 1000 Ljubljana, Slovenia

Tel: +38615437670

Fax: +38615437641

List of abbreviations: $\mathrm{Cl}$, confidence interval; $\mathrm{MM}$, malignant mesothelioma; OR, odds ratio; ROS, reactive oxygen species; RNS, reactive nitric species; SNP, single nucleotide polymorphism. 
a decreased risk of asbestosis was found for NLRP3 $\mathrm{CA}+\mathrm{AA}$ genotypes $(\mathrm{OR}=0.09,95 \% \mathrm{Cl} 0.01-0.60, \mathrm{p}=$ 0.014).

Conclusions: The results of our study suggest that NLRP3 and CARD 8 polymorphisms could affect the risk of asbestos-related diseases.

Keywords: inflammasome, polymorphism, asbestosis, pleural plaques, malignant mesothelioma

\section{Introduction}

The asbestos-related diseases, including pleural plaques, diffuse pleural thickening and pleural effusion, asbestosis, and several types of cancers, such as lung cancer, malignant mesothelioma $(M M)$ of the pleura and peritoneum, cancer of the larynx, cancer of the ovary, as well as the cancers of the buccal mucosa, the pharynx, the gastrointestinal tract, and the kidney, are still a major public health problem (14).

Pleural plaques and diffuse pleural thickening, which can be accompanied by pleural calcification, are among the most common non-malignant effects of asbestos and may occur even after relatively low asbestos exposure (5-11). Asbestosis, one of the most frequent diseases caused by asbestos, is an interstitial pulmonary process which, after a long latency period, slowly develops into diffuse pulmonary fibrosis. The disease continues to progress even after the cessation of exposure and the process is irreversible $(10,12,13)$. Among cancers, $M M$ is considered to be a highly aggressive and invasive malignoma that arises from the mesothelium, most commonly from pleura and less frequently from peritoneum or other serosal surfaces (14). As the onset of symptoms is often non-specific and insidious, this malignoma is very difficult to diagnose. However, an early diagnosis is extremely important for timely and more effective treatment (15). Therefore, potential new biomarkers for an earlier diagnosis of $M M$ have been intensively investigated $(16,17)$.

The pathogenesis of asbestos-induced pleural diseases has been extensively investigated. Asbestos fibres are thought to provoke pleural inflammation from direct toxicity to mesothelial cell. Pleural injury can be elicited by inhaled asbestos fibres indirectly via the release of inflammatory cytokines and growth factors from within the lung (18). The mechanism of cell injury caused by asbestos and affecting the cells of the pleura and lung remains unclear. It has been suggested that inflammation may have an important role in the pathogenesis of asbestos-related diseases (1921).

Inflammation has been proposed to be involved in the modification and/or calcification of pleural thickenings as well as in the development of asbestosis $(22,23)$. Chronic inflammation is also known to od azbestoze za NLRP3 CA + AA genotipove (OR =0,09, 95\% Cl 0,01-0,60, $\mathrm{p}=0,014)$.

Zaključak: Rezultati našeg istraživanja ukazuju na to da polimorfizmi NLRP3 i CARD 8 mogu uticati na rizik od bolesti povezanih sa azbestom.

Ključne reči: inflamazom, polimorfizam, azbestoza, pleuralni plakovi, maligni mezoteliom

play an essential role in tumorigenesis and is a major contributing factor in the development of many types of cancer, including $M M(22,24)$. Evidence suggests that inflammation can also be associated with unfavourable clinical prognosis in cancer patients in general (24-27) as well as in MM (28).

The reactive oxygen species (ROS) and reactive nitric species (RNS) are considered to play an important role in modulating the immune response to inflammatory stimuli (29). Recent studies have led to a better understanding of molecular mechanisms underlying the pathogenesis of asbestos-related diseases, including MM. Asbestos fibres can cause genotoxic damage either directly or indirectly, via generation of reactive oxygen and nitric species (ROS and RNS). ROS may also mediate the activation of the NLRP3 inflammasome (30).

The NLRP3 inflammasome is a multiprotein cytoplasmic complex comprised of NOD-like receptors, a family of intracellular sensors that have become known as crucial components of the innate immune responses and inflammation $(31,32)$. The NLRP3 inflammasome complex is composed of several components, such as NLRP3, CARD 8 and ASC $(23,33,34)$. It is activated by numerous physical and chemical stimuli (33), including asbestos. In response to a variety of pathogens and/or danger-associated molecular patterns, the NLRP3 inflammasome activates caspase-1, which results in IL-1 secretion and consequently inflammatory response $(33,35)$. It has been shown that the secretion of IL-1 from alveolar macrophages induced by asbestos is mediated through the NLRP3 inflammasome $(21,23)$.

It has been proposed that genetic variants of inflammasome components may influence the function of the complex and that functional polymorphisms in the NLRP3 and CARD 8 genes may be associated with IL-1 production and severe inflammation $(23,34)$.

Among the most commonly investigated inflammasome-related single nucleotide polymorphisms (SNPs) is nonsynonymous gain-of-function polymorphism NLRP3 rs35829419 (p.Gln705Lys; C > A) that leads to an overactive NLRP3 inflammasome and increased production of IL-1 (36). Another important inflammasome-related SNP is CARD 8 rs2043211 (p.Cys10Ter, A>T) that results in non-functional protein and leads to loss of CARD-8 inhibition of cas- 
pase-1 $(23,37)$. Consequently, both polymorphisms have been proposed to be associated with increased IL-1 production and proinflammatory phenotype $(21,23,36)$.

Although asbestos-related diseases are among the most frequently investigated occupational diseases and the association between asbestos exposure and asbestos-related diseases has been well proved, relatively little has been known about the genetic factors that might modify the individual susceptibility to the development of these diseases (4). This study aimed to investigate the association between NLRP3 rs35829419 and CARD 8 rs2043211 polymorphisms and the risk of developing pleural plaques, asbestosis, and $M M$, as well as to study the influence of the interactions between NLRP3 rs35829419 and CARD 8 rs2043211 polymorphisms and asbestos exposure on the risk of developing these diseases.

\section{Materials and Methods}

The case-control study included 416 subjects with pleural plaques, 160 patients with asbestosis, 154 subjects with $M M$ and 149 subjects with no asbestos disease.

Subjects with pleural plaques, asbestosis and no asbestos disease were occupationally exposed to asbestos and presented at the State Board for the Recognition of Occupational Asbestos Diseases in the period from 1 January 1998 to 31 December 2007. The diagnosis of pleural plaques, asbestosis or »no asbestos-related disease" was confirmed by two groups of experts from the State Board for the Recognition of Occupational Asbestos Diseases, each group consisting of an occupational physician, a pulmonologist, and a radiologist.

Patients with $M M$ were treated at the Institute of Oncology Ljubljana in the period between 1 January 2004 and 31 December 2012. In all patients with MM, thoracoscopy or laparoscopy/laparotomy was performed. The diagnosis of MM was histologically proved by a pathologist skilled in diagnosing this cancer.

Data on smoking were obtained for all subjects during an interview using a standardized questionnaire. The number of pack-years of smoking was calculated from the duration of smoking, and the number of cigarettes smoked per day $(38,39)$.

To determine the exposure to asbestos, a semiquantitative method was used. For the subjects with pleural plaques, asbestosis, "no asbestos-related disease " and for 33 patients with $M M$, data on cumulative asbestos exposure in fibres $/ \mathrm{cm}^{3}$-years were available from the previous study (39). Based on these data, the subjects were divided into three groups: low ( $<11$ fibres $/ \mathrm{cm}^{3}$-years), medium (11-20 fibres/ $\mathrm{cm}^{3}$-years) and high ( $>20$ fibres $/ \mathrm{cm}^{3}$-years) asbestos exposure. For patients with $M M$ with no cumulative asbestos exposure data, accurate work history was obtained, and their asbestos exposures were compared with exposures of the group of subjects with known cumulative asbestos exposure and were accordingly divided into three groups with presumed low, medium and high asbestos exposure, as previously described (40). Reliable semi-quantitative data on asbestos exposure could be obtained only for $81 \mathrm{MM}$ patients.

For the genetic analysis, genomic DNA was isolated from peripheral blood leukocytes or FTA Mini Cards (Whatman Bioscience). The NLRP3 rs35829419 and CARD 8 rs2043211 polymorphisms were determined by using a fluorescent-based competitive allele-specific polymerase chain reaction (KASPar) assay (LGC Genomics, UK) or real-time PCR-based Taqman assay (Applied Biosystems, Foster City, CA, USA) following the manufacturer's instructions.

In the statistical analysis, standard descriptive statistics was first performed, followed by univariate and multivariate logistic regression modelling. The possible interactions between genotypes as well as between genotypes and asbestos exposure were tested by logistic regression models using dummy variables. The dominant and additive genetic models were used in the analysis. All statistical analyses were performed using IBM SPSS Statistics version 21.0 (IBM Corporation, Armonk, NY, USA).

The study was approved by the Republic of Slovenia National Medical Ethics Committee and was carried out according to the Helsinki Declaration.

\section{Results}

The study included 879 subjects altogether; among them, 645 (73.4\%) were male and 234 female (26.6\%). The median age of subjects was 56.70 (inter-quartile 50.00-64.90) years. Regarding smoking, 431 (49.9\%) were ever smokers, and 432 (50.1\%) of them never smoked. Among smokers, the median number of pack-years was 19 (inter-quartile 7.3-30). Asbestos exposure was low in 538 (67.60\%) subjects, medium in 104 (13.10\%) and high in 154 (19.30\%) subjects.

The baseline characteristics of each subject group (subjects without the asbestos-related disease, patients with pleural plaques, asbestosis, MM) are presented in Table I. No statistically significant difference was found among the groups regarding gender and smoking (ever vs never smokers). On the other hand, there was a statistically significant difference considering age and asbestos exposure, as well as the pack-years among smokers. However, in all groups, the highest percentage of subjects had low asbestos exposure (Table I). 
Table I The baseline characteristics of each subject group (subjects without the disease, patients with pleural plaques, asbestosis or malignant mesothelioma).

\begin{tabular}{|c|c|c|c|c|c|c|}
\hline Characteristic & & $\begin{array}{c}\text { No disease } \\
(\mathrm{N}=149) \\
\mathrm{N}(\%)\end{array}$ & $\begin{array}{l}\text { Pleural plaques } \\
\qquad \begin{array}{c}(\mathrm{N}=416) \\
\mathrm{N}(\%)\end{array}\end{array}$ & $\begin{array}{l}\text { Asbestosis } \\
(\mathrm{N}=160) \\
\mathrm{N}(\%)\end{array}$ & $\begin{array}{c}\text { Malignant } \\
\text { mesothelioma } \\
(\mathrm{N}=154) \\
\mathrm{N}(\%)\end{array}$ & $P$ \\
\hline Gender & $\begin{array}{l}\text { Male } \\
\text { Female }\end{array}$ & $\begin{array}{l}107(71.8) \\
42(28.2)\end{array}$ & $\begin{array}{l}298(71.6) \\
118(28.4)\end{array}$ & $\begin{array}{l}121(75.6) \\
39(24.4)\end{array}$ & $\begin{array}{l}119(77.3) \\
35(22.7)\end{array}$ & $\begin{array}{c}0.486 \\
\text { Chi- } \\
\text { square }=2.444,\end{array}$ \\
\hline Age (years) & $\begin{array}{c}\text { Mean } \pm \text { SD } \\
\text { Median (25\%-75\%) } \\
\text { Min-max }\end{array}$ & \begin{tabular}{|c|}
$55.3 \pm 9.3$ \\
$53.5(47.6-62.6)$ \\
$35.4-77.8$ \\
\end{tabular} & $\begin{array}{c}55.5 \pm 9.354 .6 \\
(48.8-62.2) \\
34.5-85.8 \\
\end{array}$ & \begin{tabular}{|c|}
$58.8 \pm 9.2$ \\
$59.1(51.3-65.2)$ \\
$37.2-81.8$ \\
\end{tabular} & $\begin{array}{c}63.5 \pm 10.4 \\
65(57-70) \\
19-84\end{array}$ & $\begin{array}{c}<0.001 \\
\text { Test-statis- } \\
\text { tic }=87.357\end{array}$ \\
\hline Smoking & $\begin{array}{l}\text { No } \\
\text { Yes }\end{array}$ & $\begin{array}{l}77(51.7) \\
72(48.3)\end{array}$ & $\begin{array}{c}206(50.9)[11] \\
199(49.1)\end{array}$ & $\begin{array}{c}79(49.7)[1] \\
80(50.3)\end{array}$ & $\begin{array}{c}70(46.7)[4] \\
80(53.3)\end{array}$ & $\begin{array}{c}0.811 \\
\text { Chi- } \\
\text { square }=0.961\end{array}$ \\
\hline $\begin{array}{l}\text { Pack-years } \\
\text { (smokers only) }\end{array}$ & $\begin{array}{c}\text { Mean } \pm \text { SD } \\
\text { Median }(25 \%-75 \%) \\
\text { Min-max }\end{array}$ & $\begin{array}{l}20.7 \pm 15.0[2] \\
20(9.4-29.4) \\
0.1-65.3\end{array}$ & $\begin{array}{c}17.9 \pm 14.7[1] \\
15(5-27.8) \\
0.05-67.6\end{array}$ & $\begin{array}{c}24.7 \pm 16.7 \\
24(11.8-32.2) \\
0.15-64.5\end{array}$ & $\begin{array}{c}26.0 \pm 20.4[4] \\
20(8-40) \\
1-79.5\end{array}$ & $\begin{array}{c}0.002 \\
\text { Test-statis- } \\
\text { tic }=14.518\end{array}$ \\
\hline $\begin{array}{l}\text { Asbestos } \\
\text { exposure }\end{array}$ & $\begin{array}{l}\text { Low } \\
\text { Middle } \\
\text { High }\end{array}$ & $\begin{array}{c}122(81.9) \\
11(7.4) \\
16(10.7)\end{array}$ & $\begin{array}{c}295(72.3)[8] \\
41(10.0) \\
72(17.6)\end{array}$ & $\begin{array}{c}83(52.5)[2] \\
29(18.1) \\
46(28.8)\end{array}$ & $\begin{array}{c}38(46.9)[73] \\
23(28.4) \\
20(24.7)\end{array}$ & $\begin{array}{c}<0.001 \\
\text { Chi- } \\
\text { square }=333.371, \\
\mathrm{df}=3\end{array}$ \\
\hline
\end{tabular}

Missing data are presented in square brackets [].

P-values were calculated using the chi-square test for categorical and Kruskal-Wallis test for continuous variables.

Table II The association between baseline characteristics and asbestos-related diseases (univariate analysis).

\begin{tabular}{|c|c|c|c|c|c|c|c|c|}
\hline \multirow[t]{2}{*}{ Characteristic } & \multicolumn{2}{|c|}{$\begin{array}{l}\text { Pleural plaques } \\
\text { vs. no disease }\end{array}$} & \multicolumn{2}{|c|}{$\begin{array}{c}\text { Asbestosis vs. } \\
\text { no disease }\end{array}$} & \multicolumn{2}{|c|}{ MM vs. no disease } & \multicolumn{2}{|c|}{$\begin{array}{c}\mathrm{MM} \text { vs. } \\
\text { pleural plaques }\end{array}$} \\
\hline & OR $(95 \% \mathrm{Cl})$ & $P$ & OR $(95 \% \mathrm{Cl})$ & $P$ & OR $(95 \% \mathrm{Cl})$ & $P$ & OR $(95 \% \mathrm{Cl})$ & $P$ \\
\hline Gender & $\begin{array}{c}1.01 \\
(0.67-1.53)\end{array}$ & 0.967 & $\begin{array}{c}0.82 \\
(0.49-1.36)\end{array}$ & 0.447 & $\begin{array}{c}0.75 \\
(0.45-1.26)\end{array}$ & 0.276 & $\begin{array}{c}0.74 \\
(0.48-1.15)\end{array}$ & 0.178 \\
\hline $\begin{array}{l}\text { Age (for } 10 \\
\text { years difference) }\end{array}$ & $\begin{array}{c}1.02 \\
(0.84-1.26)\end{array}$ & 0.812 & $\begin{array}{c}1.51 \\
(1.18-1.94)\end{array}$ & 0.001 & $\begin{array}{c}2.32 \\
(1.79-3.01)\end{array}$ & $<0.001$ & $\begin{array}{c}2.37 \\
(1.91-2.93)\end{array}$ & $<0.001$ \\
\hline Smoking & $\begin{array}{c}1.03 \\
(0.71-1.50)\end{array}$ & 0.812 & $\begin{array}{c}1.08 \\
(0.69-1.69)\end{array}$ & 0.727 & $\begin{array}{c}1.22 \\
(0.78-1.92)\end{array}$ & 0.386 & $\begin{array}{c}1.18 \\
(0.81-1.72)\end{array}$ & 0.380 \\
\hline $\begin{array}{l}\text { Pack-years } \\
\text { (smokers only, } \\
\text { for } 10 \text { pack-year } \\
\text { difference) }\end{array}$ & $\begin{array}{c}0.88 \\
(0.74-1.06)\end{array}$ & 0.172 & $\begin{array}{c}1.18 \\
(0.96-1.45)\end{array}$ & 0.127 & $\begin{array}{c}1.18 \\
(0.98-1.42)\end{array}$ & 0.081 & $\begin{array}{c}1.32 \\
(1.13-1.54)\end{array}$ & 0.001 \\
\hline $\begin{array}{l}\text { Asbestos exposure } \\
\text { (medium and high } \\
\text { vs. low) }\end{array}$ & $\begin{array}{c}1.73 \\
(1.08-2.77)\end{array}$ & 0.022 & $\begin{array}{c}4.08 \\
(2.43-6.87)\end{array}$ & $<0.001$ & $\begin{array}{c}5.11 \\
(2.80-9.3)\end{array}$ & $<0.001$ & $\begin{array}{c}2.95 \\
(1.81-4.81)\end{array}$ & $<0.001$ \\
\hline
\end{tabular}

In univariate logistic regression analysis, a positive association was observed between age and asbestosis $(\mathrm{OR}=1.51,95 \% \mathrm{Cl} 1.18-1.94, \mathrm{p}=$ $0.001)$, and between age and MM (OR $=2.32,95 \%$ $\mathrm{Cl} 1.79-3.01, \mathrm{p}<0.001)$. When considering patients with $M M$ as cases and subjects with pleural plaques as controls, an increased risk of MM was found for pack-years of smoking $(\mathrm{OR}=1.32,95 \% \mathrm{Cl} 1.13$ $1.54, \mathrm{p}=0.001)$ (Table II). Asbestos exposure (medi- um and high vs low) was associated with the risk of each studied asbestos-related disease (Table II).

The genotype frequencies for NLRP3 rs35829419 and CARD 8 rs2043211 polymorphisms are shown in Table III.

The results of univariate analysis of the association between different asbestos-related diseases and NLRP3 rs35829419 and CARD 8 rs2043211 geno- 
Table III Genotype frequencies of investigated polymorphisms in asbestos-exposed subjects.

\begin{tabular}{|l|c|c|c|c|c|c|}
\hline Polymorphism & Genotype & $\begin{array}{c}\text { All subjects } \\
(\mathrm{N}=879) \\
\mathrm{N}(\%)\end{array}$ & $\begin{array}{c}\text { No disease } \\
(\mathrm{N}=149) \\
\mathrm{N}(\%)\end{array}$ & $\begin{array}{c}\text { Pleural plaques } \\
(\mathrm{N}=416) \\
\mathrm{N}(\%)\end{array}$ & $\begin{array}{c}\text { Asbestosis } \\
(\mathrm{N}=160) \\
\mathrm{N}(\%)\end{array}$ & $\begin{array}{c}\text { Malignant } \\
\text { mesothelioma } \\
(\mathrm{N}=154)\end{array}$ \\
\hline $\begin{array}{l}\text { CARD8 } \\
\text { rs2043211 }\end{array}$ & AA & $389(44.5)[4]$ & $74(50.0)$ & $167(40.3)[2]$ & $74(46.5)[1]$ & $74(48.1)$ \\
\hline p.Cys10Ter & AT & $382(43.7)$ & $57(38.5)$ & $190(45.9)$ & $68(42.8)$ & $67(43.5)$ \\
\hline $\begin{array}{l}\text { NLRP3 } \\
\text { rs35829419 }\end{array}$ & TT & $104(11.9)$ & $17(11.5)$ & $57(13.8)$ & $17(10.7)$ & $13(8.4)$ \\
\hline p.Gln705Lys & CA & $885(89.7)[4]$ & $133(89.9)[1]$ & $375(90.4)[1]$ & $145(90.6)$ & $132(86.8)[2]$ \\
\hline & AA & $2(0.2)$ & $15(10.1)$ & $39(9.4)$ & $14(8.8)$ & $20(13.2)$ \\
\hline
\end{tabular}

OMissing data are presented in square brackets [].

Table IV The association between different asbestos-related diseases and genotypes in univariate analysis.

\begin{tabular}{|c|c|c|c|c|c|c|c|c|c|}
\hline \multirow[t]{2}{*}{ Polymorphism } & \multirow[t]{2}{*}{ Genotype } & \multicolumn{2}{|c|}{$\begin{array}{c}\text { Pleural plaques vs } \\
\text { no disease }\end{array}$} & \multicolumn{2}{|c|}{$\begin{array}{l}\text { Asbestosis vs } \\
\text { no disease }\end{array}$} & \multicolumn{2}{|c|}{$\begin{array}{c}\text { MM vs } \\
\text { no disease }\end{array}$} & \multicolumn{2}{|c|}{$\begin{array}{c}\text { MM vs } \\
\text { pleural plaques }\end{array}$} \\
\hline & & OR $(95 \% \mathrm{Cl})$ & $\mathrm{P}$ & OR $(95 \% \mathrm{Cl})$ & $\mathrm{P}$ & OR $(95 \% \mathrm{Cl})$ & $P$ & OR $(95 \% \mathrm{Cl})$ & $P$ \\
\hline $\begin{array}{l}\text { CARD8 } \\
\text { rs2043211 }\end{array}$ & AA & reference & & reference & & reference & & reference & \\
\hline p.Cys10Ter & AT & $\begin{array}{c}1.48 \\
(0.99-2.21)\end{array}$ & 0.058 & $\begin{array}{c}1.19 \\
(0.74-1.92)\end{array}$ & 0.469 & $\begin{array}{c}1.18 \\
(0.73-1.90)\end{array}$ & 0.508 & $\begin{array}{c}0.80 \\
(0.54-1.18)\end{array}$ & 0.252 \\
\hline & TT & $\begin{array}{c}1.49 \\
(0.81-2.73)\end{array}$ & 0.201 & $\begin{array}{c}1.00 \\
(0.48-2.11)\end{array}$ & 1.000 & $\begin{array}{c}0.77 \\
(0.35-1.69)\end{array}$ & 0.506 & $\begin{array}{c}0.52 \\
(0.27-1.00)\end{array}$ & 0.049 \\
\hline & $\mathrm{AT}+\mathrm{TT}$ & $\begin{array}{c}1.48 \\
(1.01-2.16)\end{array}$ & 0.042 & $\begin{array}{c}1.15 \\
(0.73-1.80)\end{array}$ & 0.545 & $\begin{array}{c}1.08 \\
(0.69-1.70)\end{array}$ & 0.735 & $\begin{array}{c}0.73 \\
(0.50-1.06)\end{array}$ & 0.099 \\
\hline $\begin{array}{l}\text { NLRP3 } \\
\text { rs35829419 }\end{array}$ & CC & reference & & reference & & reference & & reference & \\
\hline p.Gln705Lys & $\mathrm{CA}+\mathrm{AA}$ & $\begin{array}{c}0.95 \\
(0.51-1.77)\end{array}$ & 0.861 & $\begin{array}{c}0.92 \\
(0.43-1.95)\end{array}$ & 0.822 & $\begin{array}{c}1.34 \\
(0.66-2.74)\end{array}$ & 0.416 & $\begin{array}{c}1.42 \\
(0.80-2.52)\end{array}$ & 0.229 \\
\hline
\end{tabular}

Table V Multiplicative interaction between CARD 8 rs2043211 and NLRP3 rs35829419 genotypes and between genotypes and asbestos exposure.

\begin{tabular}{|c|c|c|c|c|c|c|c|c|}
\hline & \multicolumn{2}{|c|}{$\begin{array}{l}\text { Pleural plaques vs } \\
\text { no disease }\end{array}$} & \multicolumn{2}{|c|}{$\begin{array}{l}\text { Asbestosis vs } \\
\text { no disease }\end{array}$} & \multicolumn{2}{|c|}{$\begin{array}{l}M M \text { vs. } \\
\text { no disease }\end{array}$} & \multicolumn{2}{|c|}{$\begin{array}{c}\text { MM vs } \\
\text { pleural plaques }\end{array}$} \\
\hline & OR $(95 \% \mathrm{Cl})$ & $\mathrm{P}$ & $\begin{array}{c}\text { OR } \\
(95 \% \mathrm{Cl})\end{array}$ & $P$ & OR $(95 \% \mathrm{Cl})$ & $\mathrm{P}$ & OR $(95 \% \mathrm{Cl})$ & $P$ \\
\hline $\begin{array}{l}\text { CARD } 8 \text { rs2043211 and } \\
\text { NLRP3 rs35829419 (both } \\
\text { dominant models) }\end{array}$ & $\begin{array}{c}0.74 \\
(0.21-2.66)\end{array}$ & 0.647 & $\begin{array}{c}0.41 \\
(0.09-1.91)\end{array}$ & 0.254 & $\begin{array}{c}0.37 \\
(0.09-1.58)\end{array}$ & 0.179 & $\begin{array}{c}0.50 \\
(0.16-1.61)\end{array}$ & 0.245 \\
\hline $\begin{array}{c}\text { CARD } 8 \text { rs } 2043211 \text { and } \\
\text { asbestos exposure }\end{array}$ & $\begin{array}{c}0.58 \\
(0.22-1.49)\end{array}$ & 0.256 & $\begin{array}{c}0.63 \\
(0.22-1.79)\end{array}$ & 0.382 & $\begin{array}{c}0.75 \\
(0.22-2.52)\end{array}$ & 0.638 & $\begin{array}{c}1.30 \\
(0.49-3.46)\end{array}$ & 0.601 \\
\hline $\begin{array}{c}\text { NLRP3 rs35829419 and } \\
\text { asbestos exposure }\end{array}$ & $\begin{array}{c}0.37 \\
(0.09-1.62)\end{array}$ & 0.188 & $\begin{array}{c}0.09 \\
(0.01-0.60)\end{array}$ & 0.014 & $\begin{array}{c}0.62 \\
(0.11-3.62)\end{array}$ & 0.591 & $\begin{array}{c}1.66 \\
(0.37-7.51)\end{array}$ & 0.513 \\
\hline
\end{tabular}


Table VI The association between different asbestos-related diseases and genotypes in multivariate analyses.

\begin{tabular}{|c|c|c|c|c|c|c|c|c|c|}
\hline \multirow[t]{2}{*}{ Polymorphism } & \multirow[t]{2}{*}{ Genotype } & \multicolumn{2}{|c|}{$\begin{array}{c}\text { Pleural plaques vs } \\
\text { no disease }\end{array}$} & \multicolumn{2}{|c|}{$\begin{array}{c}\text { Asbestosis vs } \\
\text { no disease }\end{array}$} & \multicolumn{2}{|c|}{ MM vs no disease } & \multicolumn{2}{|c|}{$M M$ vs plaques } \\
\hline & & $\begin{array}{c}\text { OR } \\
(95 \% \mathrm{Cl})\end{array}$ & $P$ & $\begin{array}{c}\text { OR } \\
(95 \% \mathrm{Cl})\end{array}$ & $P$ & $\begin{array}{c}\text { OR } \\
(95 \% \mathrm{Cl})\end{array}$ & $\mathrm{P}$ & $\begin{array}{c}\text { OR } \\
(95 \% \mathrm{Cl})\end{array}$ & $P$ \\
\hline $\begin{array}{l}\text { CARD8 } \\
\text { rs2043211 }\end{array}$ & $\mathrm{AA}$ & reference & & reference & & reference & & reference & \\
\hline \multirow[t]{3}{*}{ p.Cys10Ter } & AT & $\begin{array}{c}1.47 \\
(0.98-2.20)\end{array}$ & 0.064 & $\begin{array}{c}1.19 \\
(0.71-1.99)\end{array}$ & 0.517 & $\begin{array}{c}0.91 \\
(0.47-1.77)\end{array}$ & 0.776 & $\begin{array}{c}0.67 \\
(0.39-1.14)\end{array}$ & 0.137 \\
\hline & $\mathrm{TT}$ & $\begin{array}{c}1.42 \\
(0.77-2.62)\end{array}$ & 0.260 & $\begin{array}{c}1.14 \\
(0.51-2.57)\end{array}$ & 0.752 & $\begin{array}{c}0.61 \\
(0.19-1.94)\end{array}$ & 0.403 & $\begin{array}{c}0.37 \\
(0.14-0.96)\end{array}$ & 0.040 \\
\hline & $\mathrm{AT}+\mathrm{TT}$ & $\begin{array}{c}1.46 \\
(1.00-2.13)\end{array}$ & 0.052 & $\begin{array}{c}1.18 \\
(0.72-1.92)\end{array}$ & 0.513 & $\begin{array}{c}0.84 \\
(0.45-1.58)\end{array}$ & 0.595 & $\begin{array}{c}0.59 \\
(0.36-0.99)\end{array}$ & 0.044 \\
\hline $\begin{array}{l}\text { NLRP3 } \\
\text { rs35829419 }\end{array}$ & $\mathrm{CC}$ & reference & & reference & & reference & & reference & \\
\hline p.Gln705Lys & $\mathrm{CA}+\mathrm{AA}$ & $\begin{array}{c}0.96 \\
(0.51-1.79)\end{array}$ & 0.887 & $\begin{array}{c}1.10 \\
(0.49-2.51)\end{array}$ & 0.813 & $\begin{array}{c}0.92 \\
(0.35-2.46)\end{array}$ & 0.874 & $\begin{array}{c}1.50 \\
(0.70-3.21)\end{array}$ & 0.301 \\
\hline
\end{tabular}

Pleural plaques vs no disease: adjusted for asbestos exposure

Asbestosis vs no disease, MM vs no disease: adjusted for asbestos exposure, age, gender

MM vs plaques: adjusted for asbestos exposure, age

Table VII Multiplicative interaction between NLRP3 rs35829419 and asbestos exposure - asbestosis vs no disease.

\begin{tabular}{|c|c|c|c|c|c|c|c|c|c|c|}
\hline \multicolumn{9}{|c|}{ Asbestos exposure } & \multirow{2}{*}{\multicolumn{2}{|c|}{$\begin{array}{l}\text { Asbestos exposure } \\
\text { within NLRP3 }\end{array}$}} \\
\hline & \multicolumn{4}{|c|}{ Low } & \multicolumn{4}{|c|}{ Medium and high } & & \\
\hline $\begin{array}{c}\text { NLRP3 } \\
\text { rs358294 } \\
19\end{array}$ & $\begin{array}{c}\text { No } \\
\text { disease } \\
(\mathrm{N})\end{array}$ & $\begin{array}{c}\text { Asbestosis } \\
(\mathrm{N})\end{array}$ & $\begin{array}{c}\text { OR } \\
(95 \% \mathrm{Cl})\end{array}$ & $P$ & $\begin{array}{c}\text { No } \\
\text { disease } \\
(\mathrm{N}) \\
\end{array}$ & $\begin{array}{c}\text { Asbestosis } \\
(\mathrm{N})\end{array}$ & OR $(95 \% \mathrm{Cl})$ & $\mathrm{P}$ & OR $(95 \% \mathrm{Cl})$ & $P$ \\
\hline $\mathrm{CC}$ & 110 & 70 & 1 & reference & 23 & 73 & $\begin{array}{c}4.99 \\
(0.86-8.70)\end{array}$ & $<0.001$ & $\begin{array}{c}4.99 \\
(0.86-8.70)\end{array}$ & $<0.001$ \\
\hline $\mathrm{CA}+\mathrm{AA}$ & 11 & 13 & $\begin{array}{c}1.86 \\
(0.79-4.38)\end{array}$ & 0.157 & 4 & 2 & $\begin{array}{c}0.79 \\
(0.14-4.40)\end{array}$ & 0.274 & $\begin{array}{c}0.42 \\
(0.07-2.77)\end{array}$ & 0.369 \\
\hline $\begin{array}{c}\text { NLRP3 } \\
\text { within } \\
\text { asbestos } \\
\text { exposure }\end{array}$ & & & $\begin{array}{c}1.86 \\
(0.79-4.38)\end{array}$ & 0.157 & & & $\begin{array}{c}0.16 \\
(0.03-0.92)\end{array}$ & 0.040 & & \\
\hline
\end{tabular}

types are shown in Table IV. An increased risk of pleural plaques was found for the rs2043211 AT + TT genotypes $(\mathrm{OR}=1.48,95 \% \mathrm{Cl} 1.01-2.16, \mathrm{p}=$ $0.042)$. On the other hand, when the analysis was done for $M M$ patients as cases, and pleural plaques patients as controls, a decreased risk of $M M$ was found for carriers of TT genotype (OR $=0.52,95 \%$ $\mathrm{Cl}$ 0.27-1.00, $\mathrm{p}=0.049$ ). No association was found either between other asbestos-related diseases and CARD 8 rs2043211 genotypes or between NLRP3 rs35829419 and different asbestos-related diseases (Table IV). In the subsequent forward conditional logistic regression analysis, the results did not change considerably after adjustment for asbestos exposure, age in and gender (Table VI).
The interactions between NLRP3 rs35829419 and CARD 8 rs2043211 genotypes did not influence the risk of any asbestos-related disease. However, when testing interactions between SNPs and asbestos exposure, a multiplicative interaction was observed with NLRP3 rs35829419 (OR = 0.09, Cl 0.01-0.60, $\mathrm{p}=0.014$ ) (Table V). A decreased risk of asbestosis was found for NLRP3 CA + AA genotypes only in subjects with medium or high asbestos exposure (OR $=0.16, \mathrm{Cl}$ 0.03-0.92, $\mathrm{p}=0.040$ ) (Table VII). 


\section{Discussion}

It has been suggested that in addition to asbestos exposure, genetic factors may also affect the development of asbestos-related diseases (40-42). This study investigated the influence of NLRP3 rs35829419 and CARD 8 rs2043211 polymorphisms on the risk of different asbestos-related diseases.

As expected, asbestos exposure (medium and high vs low) was associated with an increased risk of developing all asbestos-related diseases studied, which is in agreement with the results of previous studies $(18,43-46)$.

An important finding of this study is that subjects with CARD 8 rs2043211 AT + TT genotypes had an increased risk of pleural plaques compared to those with rs2043211 AA genotype. This could be explained by observing that $A$ to $T$ transversion in CARD 8 gene can introduce a stop codon at codon 10 (Cys10Stop) and produce a truncated CARD protein, which is unable to suppress NF-kB activity. This results in an increased production of pro-IL-1 and increased inflammation $(21,23,36,47,48)$. Considering that asbestos fibres may provoke pleural inflammation, this result could be biologically plausible (18). According to our knowledge and available literature, the association between CARD 8 rs2043211 polymorphism and pleural plaques has not been studied yet.

Another interesting result of the current study shows a decreased risk of developing $M M$ compared to those patients with pleural plaques who had CARD 8 rs2043211 TT genotype, which indicates a protective effect. As described above, the activity of truncated CARD 8 protein is decreased, thus facilitating the inflammation response $(23,37)$ and consequently the increased risk of developing $M M$, which seems to be contrary to our findings. However, our results are in agreement with studies investigating the association between CARD 8 rs2043211 genotypes and other diseases $(48,49)$. Moreover, the investigated SNP represents only a fraction of the polymorphic content of the CARD8 gene (48). Therefore, other

\section{References}

1. Stayner L, Welch LS, Lemen R. The worldwide pandemic of asbestos-related diseases. Annu Rev Public Health 2013; 34: 205-16.

2. Vainio $H$. Epidemics of asbestos-related diseases-something old, something new. Scand J Work Environ Health 2015; 41: 1-4.

3. Ying SB, Jiang ZQ, He XL, Yu M, Chen RP, Chen JQ, et al. Serum HMGB1 as a Potential Biomarker for Patients with Asbestos-Related Diseases. Dis Markers 2017: 5756102.

4. Dolzan V, Dodic-Fikfak M, Franko A. Gene-Environment Interactions: The Case of Asbestosis. In: Korhan O, edi-
SNPs that may be responsible for the altered CARD8 function need to be further investigated. As NLRP3 inflammasome is involved in apoptosis (49), a decreased activity of CARD 8 protein may decrease apoptosis. Considering that apoptosis is a key part of the innate tumour-suppression mechanism (50), this could explain the decreased risk of MM for CARD8 rs2043211 TT genotype.

This study also revealed that NLRP3 polymorphism could modify the association between asbestos exposure and asbestosis. A decreased risk of asbestosis was found for subjects with NLRP3 CA and AA genotype. Considering that inflammation has been proposed to be involved in the development of asbestosis and the role of NLRP3 in the inflammation process, this interaction could be logical and biologically plausible. The results of our study are in agreement also with the findings of Kukkonen et al., who reported that the NLRP3 inflammasome is important in the development of fibrotic lung disease by associating the NLRP3 rs35829419 variant allele with increased risk of asbestos-related interstitial lung fibrosis (23).

To our knowledge and available literature, the influence of NLRP3 and CARD 8 polymorphisms on the risk of asbestos-related diseases has been not studied yet. The results of the study suggest that NLRP 3 and CARD 8 polymorphisms could affect the risk of these diseases. However, further studies including more subjects and also other NLRP3 and CARD 8 polymorphisms are necessary to elucidate these associations.

Acknowledgements and funding. This work was financially supported by the Slovenian Research Agency (ARRS Grants No. P1-0170, L3-8203).

\section{Conflict of interest statement}

The authors state that they have no conflicts of interest regarding the publication of this article.

tor. Occup Health (Lond). Rijeka: InTech, 2017: 159_ 78.

5. Jakobsson K, Stromberg U, Albin M, Welinder H, Hagman L. Radiological Changes in Asbestos-Cement Workers. Occup Environ Med 1995; 52: 20-7.

6. Hillerdal G, Henderson DW. Asbestos, asbestosis, pleural plaques and lung cancer. Scand J Work Environ Health 1997; 23: 93-103.

7. American Thoracic S. Diagnosis and initial management of nonmalignant diseases related to asbestos. Am J Respir Crit Care Med 2004; 170: 691-715. 
8. Cugell DW, Kamp DW. Asbestos and the pleura: a review. Chest 2004; 125: 1103-17.

9. Clarke CC, Mowat FS, Kelsh MA, Roberts MA. Pleural plaques: a review of diagnostic issues and possible nonasbestos factors. Arch Environ Occup Health 2006; 61: 183-92.

10. Fishwick D, Barber CM. Non-malignant asbestos-related diseases: a clinical view. Clin Med (Lond) 2014; 14: 6871.

11. Maxim LD, Niebo R, Utell MJ. Are pleural plaques an appropriate endpoint for risk analyses? Inhal Toxicol 2015; 27: 321-34.

12. Peters GA, Peters BJ. Medical aspects. In: Peters GA, Peters BJ, editors. Sourcebook on asbestos diseases: medical, legal, and engineering aspects. New York, London: Garland STPM Press, 1980: B2-B6.

13. Speizer FE, Balmes JR. Environmental lung disease. In: Fauci A, Braunwald E, Kasper D, Hauser S, Longo D, Jameson J, et al., editors. Harrison's Principles of Internal Medicine, 17th Edition. New York, Chicago, San Francisco, Lisbon, London, Madrid, Mexico City, New Delhi, San Juan, Seoul, Singapore, Sydney, Toronto: McGraw Hill, 2008: 1612-4.

14. Milinković N, Ignjatović S, Šumarac Z, Majkić-Singh N. Uncertainty of measurement in laboratory medicine. J Med Biochem 2018; 37: 279-88.

15. Tomasetti M, Santarelli L. Biomarkers for early detection of malignant mesothelioma: diagnostic and therapeutic application. Cancers 2010; 2: 523-48.

16. Franko A, Dolzan V, Kovac V, Arneric N, Dodic-Fikfak M Soluble mesothelin-related peptides levels in patients with malignant mesothelioma. Dis Markers [Research Support, Non-U.S. Gov't] 2012; 32: 123-31.

17. Kovac V, Dodic-Fikfak M, Arneric N, Dolzan V, Franko A. Fibulin-3 as a biomarker of response to treatment in malignant mesothelioma. Radiol Oncol 2015; 49: 279-85.

18. Chapman SJ, Cookson WO, Musk AW, Lee YC. Benign asbestos pleural diseases. Curr Opin Pulm Med 2003; 9 . 266-71.

19. Mossman BT, Churg A. Mechanisms in the pathogenesis of asbestosis and silicosis. Am J Respir Crit Care Med 1998; 157: 1666-80.

20. Kamp DW, Weitzman SA. The molecular basis of asbestos induced lung injury. Thorax 1999; 54: 638-52.

21. Dostert C, Petrilli V, Van Bruggen R, Steele C, Mossman BT, Tschopp J. Innate immune activation through Nalp3 inflammasome sensing of asbestos and silica. Science 2008; 320: 674-7.

22. Matsuzaki H, Maeda M, Lee S, Nishimura $Y$, KumagaiTakei N, Hayashi $\mathrm{H}$, et al. Asbestos-Induced Cellular and Molecular Alteration of Immunocompetent Cells and Their Relationship with Chronic Inflammation and Carcinogenesis. J Biomed Biotechnol 2012: 492608.

23. Kukkonen MK, Vehmas T, Piirila P, Hirvonen A. Genes involved in innate immunity associated with asbestosrelated fibrotic changes. Occup Environ Med 2014; 71: 48-54.
24. Kadariya Y, Menges CW, Talarchek J, Cai KQ, KleinSzanto AJ, Pietrofesa RA, et al. Inflammation-Related IL1 beta/IL1R Signaling Promotes the Development of Asbestos-Induced Malignant Mesothelioma. Cancer Prev Res 2016; 9: 406-14.

25. Leek RD, Lewis CE, Whitehouse R, Greenall M, Clarke J Harris AL. Association of macrophage infiltration with angiogenesis and prognosis in invasive breast carcinoma. Cancer Res 1996; 56: 4625-9.

26. Ribatti D, Ennas MG, Vacca A, Ferreli F, Nico B, Orru S et al. Tumor vascularity and tryptase-positive mast cells correlate with a poor prognosis in melanoma. Eur J Clin Invest 2003; 33: 420-5.

27. Dunn JH, Ellis LZ, Fujita M. Inflammasomes as molecular mediators of inflammation and cancer: Potential role in melanoma. Cancer Lett 2012; 314: 24-33.

28. Comar M, Zanotta N, Bonotti A, Tognon M, Negro C, Cristaudo A, et al. Increased Levels of C-C Chemokine RANTES in Asbestos Exposed Workers and in Malignant Mesothelioma Patients from an Hyperendemic Area. PloS one 2014; 9: e104848.

29. Wu YZ, Antony S, Meitzler JL, Doroshow JH. Molecular mechanisms underlying chronic inflammation-associated cancers. Cancer Lett 2014; 345: 164-73.

30. Chew SH, Toyokuni S. Malignant mesothelioma as an oxidative stress-induced cancer: An update. Free Radic Biol Med 2015; 86: 166-78.

31. Petrilli V, Dostert C, Muruve DA, Tschopp J. The inflammasome: a danger sensing complex triggering innate immunity. Curr Opin Immunol 2007; 19: 615-22.

32. Westbom C, Thompson JK, Leggett A, MacPherson $M$, Beuschel S, Pass $\mathrm{H}$, et al. Inflammasome Modulation by Chemotherapeutics in Malignant Mesothelioma. PloS one 2015; 10: e0145404.

33. Gross O, Thomas CJ, Guarda G, Tschopp J. The inflammasome: an integrated view. Immunol Rev 2011; 243 : 136-51.

34. Verma D, Lerm M, Blomgran JR, Eriksson $P$, Soderkvist $P$, Sarndahl E. Gene polymorphisms in the NALP3 inflammasome are associated with interleukin-1 production and severe inflammation: relation to common inflammatory diseases? Arthritis Rheum 2008; 58: 888-94.

35. da Silva WC, Oshiro TM, de Sa DC, Franco DDGS, Neto CF, Pontillo A. Genotyping and differential expression analysis of inflammasome genes in sporadic malignant melanoma reveal novel contribution of CARD8, IL1B and IL18 in melanoma susceptibility and progression. Cancer Genet-Ny 2016; 209: 474-80.

36. Verma D, Sarndahl E, Andersson H, Eriksson P, Fredrikson $M$, Jonsson Jl, et al. The Q705K Polymorphism in NLRP3 Is a Gain-of-Function Alteration Leading to Excessive Interleukin-1 beta and IL-18 Production. PloS one 2012; 7: e34977.

37. Fontalba A, Martinez-Taboada V, Gutierrez O, Pipaon C, Benito N, Balsa A, et al. Deficiency of the NF-kappa B inhibitor caspase activating and, recruitment domain 8 in patients with rheumatoid arthritis is associated with disease severity. J Immunol 2007; 179: 4867-73. 
38. Ferris BG. Epidemiology Standardization Project. Am Rev Respir Dis 1978; 118: 1-120.

39. Fikfak MD, Kriebel D, Quinn MM, Eisen EA, Wegman $\mathrm{DH}$. A case control study of lung cancer and exposure to chrysotile and amphibole at a Slovenian asbestos-cement plant. Ann Occup Hyg 2007; 51: 261-8.

40. Franko A, Kotnik N, Goricar K, Kovac V, Dodic-Fikfak M, Dolzan V. The Influence of Genetic Variability on the Risk of Developing Malignant Mesothelioma. Radiol Oncol 2018; 52: 105-11.

41. Bhattacharjee P, Paul S, Bhattacharjee P. Risk of occupational exposure to asbestos, silicon and arsenic on pulmonary disorders: Understanding the genetic-epigenetic interplay and future prospects. Environ Res 2016; 147: 425-34.

42. Franko A, Dolžan $\mathrm{V}$, Arneri $\mathrm{N}$, Dodič-Fikfak $M$. The influence of gene-gene and gene-environment interactions on the risk of asbestosis. Biomed Res Int 2013; 2013: 405743.

43. Mossman BT, Gee JB. Asbestos-related diseases. N Engl J Med 1989; 320: 1721-30.

44. Jamrozik E, de Klerk N, Musk AW. Asbestos-related disease. Intern Med J 2011; 41: 372-80.
45. Frank AL, Joshi TK. The global spread of asbestos. Annals of global health 2014; 80: 257-62.

46. Straif K, Benbrahim-Tallaa L, Baan R, Grosse Y, Secretan $B$, El Ghissassi F, et al. A review of human carcinogensPart C: metals, arsenic, dusts, and fibres. The Lancet Oncology 2009; 10: 453-4.

47. Vasseur F, Sendid B, Broly F, Gower-Rousseau C, Sarazin A, Standaert-Vitse A, et al. The CARD8 p.C10X mutation associates with a low anti-glycans antibody response in patients with Crohn's disease. BMC Med Genet 2013; 14: 35 .

48. Zhang ZT, Ma XJ, Zong Y, Du XM, Hu JH, Lu GC. Is the CARD8 rs2043211 polymorphism associated with susceptibility to Crohn's disease? A meta-analysis. Autoimmunity 2015; 48: 524-31.

49. Ito $S$, Hara $Y$, Kubota T. CARD8 is a negative regulator for NLRP3 inflammasome, but mutant NLRP3 in cryopyrin-associated periodic syndromes escapes the restriction. Arthrit Res Ther 2014; 16: R52.

50. Kaczanowski S. Apoptosis: its origin, history, maintenance and the medical implications for cancer and aging. Phys Biol 2016; 13: 031001. 